\title{
Thermal noise study of a radiation pressure noise limited optical cavity with fused silica mirror suspensions ${ }^{\star}$
}

\author{
Sibilla Di Pace ${ }^{1,2, a}$, Luca Naticchioni ${ }^{2}$, Martina De Laurentis ${ }^{3}$, and Flavio Travasso ${ }^{4}$ \\ 1 Università di Roma La Sapienza, Rome, Italy \\ 2 INFN Sezione di Roma, Rome, Italy \\ 3 Università di Napoli Federico II \& INFN Sezione di Napoli, Naples, Italy \\ 4 Università di Camerino \& INFN Sezione di Perugia, Perugia, Italy
}

Received 30 March 2020 / Received in final form 1 July 2020 / Accepted 22 September 2020 Published online 1 November 2020

(C) The Author(s) 2020. This article is published with open access at Springerlink.com

\begin{abstract}
In this work we study the thermal noise of two monolithically suspended mirrors in a tabletop high-finesse optical cavity. We show that, given suitable seismic filters, such a cavity can be designed to be sensitive to quantum radiation pressure fluctuations in the audio band of gravitational wave interferometric detectors below $1 \mathrm{kHz}$. Indeed, the thermal noise of the suspensions and of the coatings constitutes the main limit to the observation of quantum radiation pressure fluctuations. This limit can be overcome with an adequate choice of mirror suspension and coating parameters. Finally, we propose to combine two optical cavities, like those modeled in this work, to obtain a tabletop quantum radiation pressure-limited interferometer.
\end{abstract}

\section{Introduction}

Thermal noise places a limit for all the experiments designed to measure small position fluctuations. This is the case of gravitational wave interferometers, in particular in a band ranging from few to some hundreds of $\mathrm{Hz}$. Another limitation to free-mass position measurements in interferometric experiments at low frequency comes from the quantum radiation pressure noise. This effect has been recently observed in interferometric gravitational wave detectors like Virgo [1,2] and LIGO [3]. In particular, the combination of quantum radiation pressure fluctuations at low frequencies and shot noise at higher frequencies introduces an intrinsic limit to the measurement process, called Standard Quantum Limit (SQL) $[4,5]$, due to the quantum nature of light. However, the effect of radiation pressure can be also exploited in optomechanical cavities to generate a quantum correlation between phase and amplitude noises, producing the so-called ponderomotive squeezing [6]. In this paper, we will consider an optical cavity with suspended mirrors having different masses, where the lighter one behaves like an optical spring. Then, we propose to combine two identical optomechanical cavities of this kind obtaining an interferometric configuration, thus allowing to cancel out all the noises common to the two arm-cavities, like e.g. the RIN. In this configuration, given a proper seismic isolation, pendulum thermal

\footnotetext{
* Contribution to the Topical Issue "Quantum Technologies for Gravitational Physics", edited by Tanja Mehlstäubler, Yanbei Chen, Guglielmo M. Tino, Hsien-Chi Yeh.

${ }^{a}$ e-mail: sibilla.dipace@roma1.infn.it
}

noise constitutes the main disturbance which is more likely to be dominant, thus preventing to achieve the quantum radiation pressure limit. For this reason, we have studied in detail possible configurations of the system, evaluating a range of key parameters and the experimental feasibility aspects. In particular, we propose to adopt a monolithic suspension for the cavity mirrors, finding a suitable choice of parameters for which the thermal noise is low enough to allow the observation of the quantum radiation pressure effect in the audio low-frequency range $(10 \mathrm{~Hz}-$ $1 \mathrm{kHz}$ ). With this preliminary study we demonstrate the feasibility of a tabletop experiment aiming at producing frequency dependent ponderomotive squeezing that could be exploited for broadband quantum noise reduction in future generation of gravitational wave detectors.

\section{Optical spring in a detuned cavity}

Let us consider the case of a Fabry-Pérot cavity of length $L$, tuned close to the cavity resonance frequency $\omega_{r}$, and composed of a fixed and high-reflective input mirror with mass $M$ and a suspended perfectly reflective end mirror with mass $m$. The impinging coherent laser beam has frequency $\omega_{0}$ and input power $I_{0}$. The power circulating inside the Fabry-Pérot cavity is thus:

$$
W\left(I_{0}, \phi\right)=I_{0} G \frac{1}{1+F \sin ^{2}\left(\frac{\phi}{2}\right)}
$$

where $\phi$ is the phase of the light field inside the cavity, $G$ is the gain of the Fabry-Pérot cavity related to the 
finesse $\mathcal{F}$ of the cavity by $G=2 \mathcal{F} / \pi$ and $F=G^{2}$ is the coefficient of finesse. The phase $\phi$ is: $\phi=\delta \omega / \Delta \nu$ where $\Delta \nu$ is the free spectral range of the cavity, $c$ is the speed of light and $\delta \omega=\left(\omega_{r}-\omega_{0}\right)$ is the detuning factor of this cavity [7]. We now introduce the linewidth [7]:

$$
\gamma=\frac{c T_{i n}}{4 L}
$$

where $T_{\text {in }}$ is the input mirror transmittivity. Then, if we rewrite the term $F \sin ^{2}\left(\frac{\phi}{2}\right)$, considering the approximation on the phase term $\sin \left(\frac{\phi}{2}\right) \approx\left(\frac{\phi}{2}\right)$, we obtain that:

$$
F\left(\frac{\phi}{2}\right)^{2}=\left(\frac{\delta \omega}{\gamma}\right)^{2}
$$

We can now define the normalized detuning $\delta_{\gamma}$ as [7]:

$$
\delta_{\gamma}=\frac{\delta \omega}{\gamma}=\frac{4 L\left(\omega_{r}-\omega_{0}\right)}{c T_{i n}}
$$

Then, taking into account equations (1), (3) and (4), we can finally write the power circulating inside the cavity as $[7]$ :

$$
W\left(I_{0}, \delta_{\gamma}\right)=I_{0} \frac{2 \mathcal{F}}{\pi} \frac{1}{1+\delta_{\gamma}^{2}} .
$$

In such an optomechanical system, the joint action of radiation pressure and gravitational force can be described as a spring with high stiffness acting on the end mirror when the cavity length fluctuates. The radiation pressure force, i.e. the ponderomotive force, acting on the suspended mirror depends on the circulating power:

$$
F_{p}=\frac{2 W}{c} .
$$

It is always positive and inversely proportional to the cavity length (i.e. to the detuning), if we consider a detuning to the long side, while the gravitational force acts as a restoring force, like in a classical pendulum. Considering the fluctuations of the mirror position $x$, those of the input power $I$, and a generic external force $F_{\text {ext }}$, the equation of motion for the suspended cavity mirror can be written in this form:

$$
-m \omega^{2} x=-k_{s} x+\frac{2}{c} \frac{\partial W}{\partial I_{0}} \bar{I}_{0}+F_{\text {ext }}
$$

where $\bar{I}_{0}$ is the average of the varying input power $I_{0}$, and

$$
k_{s}=k_{g}+k_{\mathrm{opt}}
$$

is the total spring constant containing both the gravitational $\left(k_{g}\right)$ and the optical spring $\left(k_{\text {opt }}\right)$ terms. For small displacements $k_{g}$ takes into account the gravitational component related to the pendulum of length $l$ determined by the suspended mirror of mass $m, k_{g}=\frac{m g}{l}$ (where $g$ is the gravitational acceleration). The optical spring constant, due to the radiation pressure, is defined by the first derivative of the radiation pressure force with respect to the displacement $[6,7]$ :

$$
k_{\mathrm{opt}}=-\frac{2}{c} \frac{\partial W}{\partial x} \equiv-\frac{2}{c} \frac{\partial W}{\partial \delta_{\gamma}} \frac{\partial \delta_{\gamma}}{\partial x}=-\frac{4 \omega_{0} W}{\gamma L c} \frac{\delta_{\gamma}}{1+\delta_{\gamma}^{2}} .
$$

Note that the derivative $\frac{\partial \delta_{\gamma}}{\partial x}$ is calculated considering that the cavity resonance frequency $\omega_{r}=2 \pi \Delta \nu=\frac{\pi c}{L}$. In equation (7) the term $k_{s}=k_{g}+k_{\text {opt }}$ shows the linear elastic response of the system to small displacements: if the detuning increases (i.e. the cavity becomes longer), the impinging power decreases and the mirror is pushed back; if the detuning decreases (i.e. the cavity becomes shorter), the impinging power increases and the mirror is pushed forward. This occurs for a detuning to the long side. Moreover, the optical spring constant will be $k_{\text {opt }}>0$ when the cavity is longer than the resonant length, and $k_{\text {opt }}<0$ when the cavity is shorter than that value. Finally, when $\left|k_{\text {opt }}\right|>k_{g}$, and so $k_{s}<0$, the cavity becomes unstable. The feasibility of an optomechanical quantum-correlated system, such as the optical spring, has been demonstrated in the past years with small membrane resonators in the $\mathrm{MHz}$ region [8] and more recently (2019) with microresonators [9] at lower frequencies. Moreover, the effect of this quantum behaviour has been recently observed also for $\mathrm{kg}$-scale mirrors in Advanced gravitational wave detectors $[2,3]$. Indeed, present technology assures the possibility to efficiently filter out seismic noise $[10,11]$. Then, it is possible to design a macroscopic optomechanical cavity with inch-size-scale suspended mirrors, in which the lighter mirror is in the regime dominated by the quantum radiation pressure noise. This seems feasible with a mirror suspension system tailored in such a way to keep the thermal noise associated to the suspension low enough, and using low dissipation mirror coatings. Setting up an experiment with a detuned optical cavity with suspended mirror, it is possible to design the mechanical suspension in order to have the mechanical pendulum frequency much lower than the optical spring frequency. Moreover, working in the range between the pendulum and the optical spring frequencies, for the evaluation of dominant noise contributions, as the suspension thermal noise and the radiation pressure noise, the optical spring contribution can be neglected, and a classical approach can be used.

\section{Single stage cavity mirror suspension}

In this section, we describe a possible cavity configuration where a high reflective mirror is hung through silica $\left(\mathrm{SiO}_{2}\right)$ fibers in a single stage suspension system. This material is a suitable choice since silica has high quality surface and good resistance to breakage [12]. Indeed, there is a deep expertise using silica fibers in the $\mathrm{kg}$-scale test masses suspension in gravitational wave detectors $[13,14]$. In Section 3.1.2, we evaluate the thermal noise fluctuations for different suspension configurations, given a mirror mass within the orders of magnitude $1 \mathrm{~g}-100 \mathrm{~g}$. Then, we compare these results with the quantum radiation pressure fluctuations expected in a high finesse cavity with $\mathcal{F}$ in the range of some tens of thousands. Indeed, the suspension thermal noise associated to this system must be lower than the quantum radiation pressure fluctuations in order to exploit the optical spring effect to produce squeezing. 


\subsection{Evaluation of the suspension parameters}

The model parameters of the suspension to be defined are: the length $l$, the diameter $d$ of the silica fibers and the mass $m$ of the suspended mirror. We consider $l$ ranging between $30 \mathrm{~cm}$ and $50 \mathrm{~cm}$, due to space constraints in a vacuum chamber that could host a tabletop experiment. The diameter $d$ of the silica fibers can vary from $10 \mu \mathrm{m}$, the minimal diameter experimentally achievable for few tens of cm-long fibers, to $400 \mu \mathrm{m}$, the value of those used in Advanced Virgo. For the suspended mirror mass we investigate values ranging within the orders of magnitude $1 \mathrm{~g}$ and $100 \mathrm{~g}$. Given the proposed mirror mass range, the mirror diameter can vary between 1 and 3 inches, that are substrate dimensions easily available on the market. In this section, we describe how we evaluate the thermal noise of the single stage mirror suspension given the parameters ranges described before. Then, we compare them to the quantum radiation pressure noise evaluated considering an input laser power $P_{\text {in }}=2.5 \mathrm{~W}$, a cavity finesse $\mathcal{F}$ of the order of few tens of thousands and a laser wavelength of $\lambda=1064 \mathrm{~nm}$. This preliminary analysis allows us to select those combinations of parameters which are a good trade-off for the feasibility of a quantum radiation pressure noise-limited optical cavity with suspended mirrors.

\subsubsection{Dilution factor}

The first analysis done to select among the feasible values of suspended mass $m$, suspension length $l$ and suspension diameter $d$, consists in the evaluation of the so called dilution factor $D_{i l F}$ [15]. It allows to discriminate those parameters for which the mirror suspended to the silica fibers behaves as a gravitational pendulum. In a real wire the pendulum frequency is given by:

$$
\omega_{p}^{2}=\omega_{g}^{2}+\omega_{e l}^{2}
$$

where the gravitational contribution is:

$$
\omega_{g}=\sqrt{g / l}
$$

and the elastic term is:

$$
\omega_{e l}=\sqrt{k_{e l} / m} .
$$

The elastic coefficient $k_{e l}$ in equation (12) depends on the wire geometry and material, and it is defined as [15]:

$$
k_{e l}=\sqrt{\Lambda Y J} /\left(2 l^{2}\right)
$$

where $\Lambda$ is the tension of each wire, $Y$ the Young's modulus of the wire material and $J$ the momentum of inertia of the wire section with respect to the main axis. The dilution factor is then defined as:

$$
D_{i l F}=\omega_{p}^{2} / \omega_{e l}^{2}=1+\omega_{g}^{2} / \omega_{e l}^{2} .
$$

It indicates which term dominates between the gravitational and the elastic, therefore if the suspension behaves as an ideal wire, or as an elastic body with stiffness $k_{e l}$. In
Table 1 we report a selection of the dilution factor $D_{i l F}$ calculated for several combinations of $m, l$ and $d$ values varying in the ranges described in Section 3.1. Those combinations for which the gravitational contribution is dominant (i.e. those for which $D_{i l F} \gg 1$ ) and that are a good feasibility trade-off for an experimental setup, are pointed out in bold in Table 1. These values are then further investigated with an estimation of the associated simple pendulum thermal noise. For this selection we should consider that it is not convenient to use a $1 \mathrm{~g}$ mirror, since it would require very thin suspension wires $(10-20) \mu \mathrm{m}$, whereas it seems more suitable to use a $10 \mathrm{~g}$ or a $100 \mathrm{~g}$ mirror mass.

\subsubsection{Single stage suspension thermal noise}

The second step of this analysis consists in the calculation of the simple pendulum thermal noise, hereafter referred as ThNP, for all the combinations selected in Section 3.1.1. Using the fluctuation dissipation theorem (FDT) [16] we can evaluate the power spectral density of the mirror position fluctuations due to the thermal noise, that for a simple pendulum are given by:

$$
X_{T h N P}(\omega)=\sqrt{\frac{4 k_{B} T}{m \omega} \frac{\omega_{p}^{2} \phi_{p}(\omega)}{\left(\left(\omega_{p}^{2}-\omega^{2}\right)^{2}+\left(\omega_{p}^{2} \phi_{p}(\omega)\right)^{2}\right)}}
$$

where $k_{B}$ is the Boltzmann constant, $T$ the temperature and $\phi_{p}(\omega)$ is the pendulum loss angle which takes into account all the dissipation processes in the silica fiber [15]:

$$
\phi_{p}(\omega)=D_{i l F}^{-1}\left[\phi_{S_{i O}}+\phi_{e}+\phi_{t e}(\omega)\right] .
$$

In the last definition $\phi_{\mathrm{SiO}_{2}}$ is the loss angle due to the intrinsic losses of fused silica [17]:

$$
\phi_{\mathrm{SiO}_{2}}=4.1 \times 10^{-9},
$$

and $\phi_{e}$ represents the surface losses in the dissipation depth $d_{s}$, given the surface to volume ratio $S / V$ of the fiber $[18,19]$ :

$$
\phi_{e}=\phi_{S_{i O}}\left(2 d_{s} S\right) / V .
$$

The last term of equation (16) represents the loss angle associated to the thermoelastic losses [20]:

$$
\phi_{t e}(\omega)=\Delta_{s} \frac{\left(\omega \tau_{s}\right)}{\left(1-\left(\omega \tau_{s}\right)^{2}\right)} .
$$

In equation (19) the term $\Delta_{s}$ and the characteristic time of heat diffusion $\tau_{s}$ depend on the material and geometrical properties of the suspension as follows [20]:

$$
\Delta_{s}=\frac{Y T}{\rho C}\left(\alpha-\beta \frac{\Lambda}{A Y}\right)^{2} ; \quad \tau_{s}=\frac{C \rho(2 r)^{2}}{2.16 \cdot 2 \pi \kappa}
$$

where $C, \kappa, \rho$ and $\alpha$ are respectively the specific heat, the thermal conductivity, the density and the coefficient of linear expansion of the fused silica. The second order term of $\Delta_{s}$ depends on $\beta$ which is the relative coefficient 
Table 1. The dilution factor $D_{i l F}$ calculated for several combinations of the suspension parameters: $m, l$ and $d$. Values highlighted in bold correspond to those sets of parameters representing a good trade-off for the feasibility of the proposed tabletop optical cavity, given the available silica fiber production facilities such as those located at EGO, Virgo site.

\begin{tabular}{lccccccc}
\hline$l(\mathrm{~cm})$ & $m(\mathrm{~g})$ & $d=10 \mu \mathrm{m}$ & $20 \mu \mathrm{m}$ & $50 \mu \mathrm{m}$ & $100 \mu \mathrm{m}$ & $200 \mu \mathrm{m}$ & $400 \mu \mathrm{m}$ \\
\hline 30 & 1 & $1.4 \times 10^{4}$ & $3.5 \times 10^{3}$ & $5.6 \times 10^{2}$ & $1.4 \times 10^{2}$ & 36 & 9.8 \\
40 & 1 & $1.5 \times 10^{4}$ & $4.7 \times 10^{3}$ & $7.5 \times 10^{2}$ & $1.5 \times 10^{2}$ & 48 & 13 \\
50 & 1 & $2.4 \times 10^{4}$ & $5.9 \times 10^{3}$ & $9.4 \times 10^{2}$ & $2.3 \times 10^{2}$ & 60 & 16 \\
30 & 10 & $4.5 \times 10^{4}$ & $1.1 \times 10^{4}$ & $1.8 \times 10^{3}$ & $4.5 \times 10^{2}$ & $1.1 \times 10^{2}$ & 29 \\
40 & 10 & $6.0 \times 10^{4}$ & $1.5 \times 10^{4}$ & $\mathbf{2 . 4} \times \mathbf{1 0}^{3}$ & $6.0 \times 10^{2}$ & $1.5 \times 10^{2}$ & 38 \\
50 & 10 & $7.4 \times 10^{4}$ & $1.9 \times 10^{4}$ & $3.0 \times 10^{3}$ & $7.4 \times 10^{2}$ & $1.9 \times 10^{2}$ & 48 \\
30 & 100 & $1.4 \times 10^{5}$ & $3.5 \times 10^{2}$ & $5.6 \times 10^{3}$ & $1.4 \times 10^{3}$ & $3.5 \times 10^{2}$ & 89 \\
40 & 100 & $1.9 \times 10^{5}$ & $4.7 \times 10^{4}$ & $\mathbf{7 . 5} \times \mathbf{1 0}^{3}$ & $\mathbf{1 . 9} \times \mathbf{1 0}^{3}$ & $4.7 \times 10^{2}$ & $1.2 \times 10^{2}$ \\
50 & 100 & $2.4 \times 10^{5}$ & $5.9 \times 10^{4}$ & $9.4 \times 10^{3}$ & $\mathbf{2 . 4} \times \mathbf{1 0}^{\mathbf{3}}$ & $5.9 \times 10^{2}$ & $1.4 \times 10^{2}$ \\
\hline
\end{tabular}

of thermal gradient of the Young's modulus $Y$ of the fused silica defined as:

$$
\beta=\frac{1}{Y} \frac{d Y}{d T} .
$$

In equations (20), the parameters $r, \Lambda$ and $A$ are respectively the radius, the tension and the section area of the wire. At this point, the pendulum thermal noise of each selected set of parameters can be compared with the expected quantum radiation pressure noise $(\mathrm{RPN})$ in order to validate the configuration for which the suspended mirror in the cavity is RPN-limited. Given the conditions mentioned at the end of Section 3, RPN mirror fluctuations of a simple pendulum suspension in a Fabry-Pérot cavity can be modeled following a classical approach [21]:

$$
X_{R P}(\omega)=\frac{2 \mathcal{F}}{\pi m} \sqrt{\frac{8 h_{P} P_{i n} /(\lambda c)}{\left(\omega_{p}^{2}-\omega^{2}\right)^{2}+\left(\omega_{p}^{2} \phi_{p}(\omega)\right)^{2}}}
$$

where $\lambda$ is the laser wavelength, $h_{P}$ the Planck constant, $c$ the speed of light. Assuming a finesse of $\mathcal{F}=3 \times 10^{4}$, an input laser power $P_{\text {in }}=2.5 \mathrm{~W}$ and a wavelength $\lambda=1064 \mathrm{~nm}$, we obtain the plot of Figure 1 . In this plot we compare the simple pendulum thermal noise (ThNP, continuous lines) and the quantum radiation pressure noise (RPN, dashed lines) estimated for the four combinations of parameters selected from Table 1. This second step of our analysis allows us to select two suitable configurations, one for a $10 \mathrm{~g}$ mirror (black curves) and the other for a $100 \mathrm{~g}$ mirror (red curves). For both mass values we select $40 \mathrm{~cm}$-long silica fibers with a radius of $25 \mu \mathrm{m}$. This choice is compliant with the experimental feasibility for the fiber production, assuring a significant clearance between RPN and ThNP.

\section{Double stage cavity mirror suspension}

In the previous section, a single stage-like configuration for the suspension of the cavity mirrors has been investigated, and the associated suspension thermal noise has been evaluated. This analysis, allowed to define the suspension parameters to be further investigated such as mirror mass, pendulum length and fiber diameter. Nevertheless, a double stage-like configuration seems a more

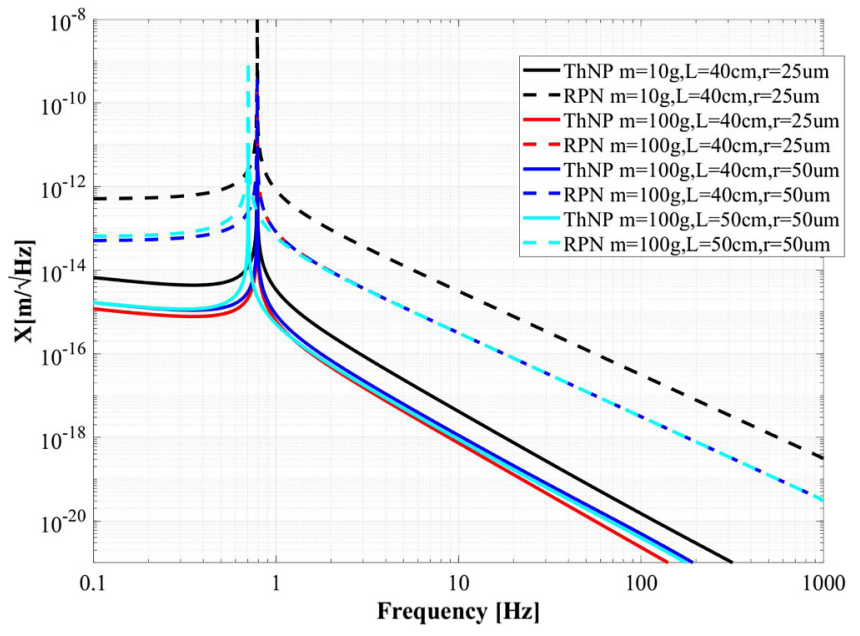

Fig. 1. Simple pendulum thermal noise (ThNP, continuous lines) compared to quantum radiation pressure fluctuations (RPN, dashed lines) for the sets of parameters selected from Table 1 . Here only the worst case (cyan curves) among the chosen ones and a similar configuration (blue curves) are compared to the configuration selected for a $10 \mathrm{~g}$ mirror (black curves) and the configuration selected for a $100 \mathrm{~g}$ mirror (red curves). RPN is evaluated assuming $\mathcal{F}=3 \times 10^{4}, P_{\text {in }}=2.5 \mathrm{~W}$ and $\lambda=1064 \mathrm{~nm}$.

suitable choice for several reasons. A double pendulum configuration for the mirror suspension gives three main advantages, since the presence of an intermediate suspension stage: (a) provides, at the level of the mirror, a further seismic noise attenuation fundamental in the first phase of the experiment, when it will not have other suspension stages (see Sect. Conclusions and [34]); (b) allows to have a double steering of the mirror, both at the level of the mirror and of the intermediate suspension stage, in order to control all degrees of freedom in a hierarchical control scheme which can provide lower noise signals; (c) allows to ease the process of integration of the monolithic suspensions which can be assembled out of the bench and then clamped from the intermediate suspension stage instead of the upper bigger bench that will be full of other elements. This double-stage configuration is there- 


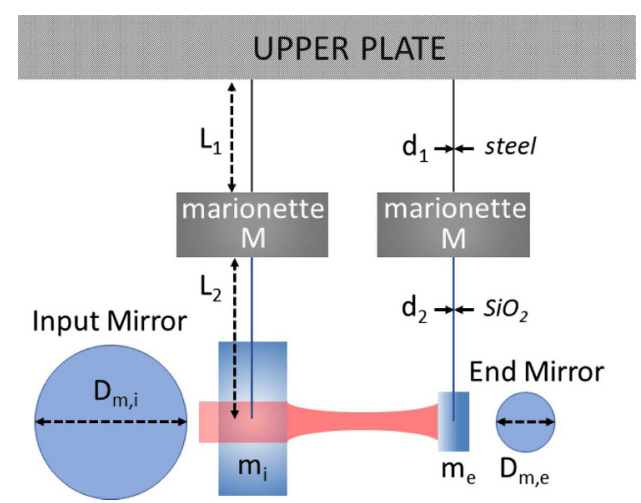

Fig. 2. Scheme of the suspended optical cavity considered in our analysis. Each mirror, suspended from an intermediate stage called marionette, can be described as a double stage pendulum. The input and end mirrors, of mass $m$ and diameter $D_{m}$, are monolithically suspended through two $\mathrm{SiO}_{2}$ fibers (length $L_{2}$, diameter $d_{2}$ ) from the marionette (mass $M$ ), which in turn is hung from an upper plate through a wire (length $L_{1}$, diameter $d_{1}$ ) made of C85 steel. The cavity length value can range between $100 \mathrm{~mm}$ and $1 \mathrm{~m}$, due to tabletop space constraints.

fore evaluated as a more reliable choice for a setup like the one presented in this work. Given that, the next step of our analysis consists in comparing the quantum radiation pressure noise with the suspension thermal noise in the case of a Fabry-Pérot cavity composed of two mirrors in a double stage suspension configuration.

\subsection{Model description}

In this model the mirrors of the Fabry-Pérot cavity are suspended in double pendulum configuration as depicted in Figure 2. In particular, each mirror is suspended by two silica fibers from an intermediate steering stage made of steel, called marionette, hung from the suspension point with a steel wire. To increase the suspension quality factor (i.e. to reduce the associated thermal noise [12]), we use the so-called monolithic suspension (see Sect. 4.2). This configuration is defined monolithic because the mirror and the fibers are made of the same material, and the two parts are attached in such a way to be considered as a single body [13]. At the top level, the fibers are welded to fused silica anchors that are in turn silicatebonded to the marionette of mass $M=1 \mathrm{~kg}$. This stage is directly suspended from an upper plate by a wire made of $C 85$ stainless steel having diameter $d_{1}=300 \mu \mathrm{m}$ and length $L_{1}=30 \mathrm{~cm}$. These values are compliant with the feasibility requirements of a tabletop experiment. According to the mirror mass values selected before, we choose two fused silica bulks: the lighter end mirror having mass $m_{e}=10 \mathrm{~g}$, which corresponds to a cylinder of diameter $D_{m, e}=1^{\prime \prime}$ and thickness $t_{e}=1 \mathrm{~cm}$, and the heavier input mirror having mass $m_{i}=300 \mathrm{~g}$. The latter value, still in the order of magnitude of hundreds of grams, corresponds to a cylinder of diameter $D_{m, i}=3^{\prime \prime}$ and thickness $t_{i}=3 \mathrm{~cm}$. Moreover, this mass value still satisfies the requirement on the dilution factor mentioned in the previous section. Indeed, in the case of a $300 \mathrm{~g}$ mirror, for the chosen silica fibers parameters, we obtain a dilution factor of $1.3 \times 10^{4}$, which is even higher than the values obtained for $100 \mathrm{~g}$ (see Tab. 1). For the length of the Fabry-Pérot cavity, we should consider values ranging between $100 \mathrm{~mm}$ and $1 \mathrm{~m}$, due to space constraints in a vacuum chamber that could host a tabletop experiment. Finally, an high reflective coating, which provides a transmissivity of $T=1 \mathrm{ppm}$ at $1064 \mathrm{~nm}$, on the end mirror intra-cavity surface, is needed to reach the required value of finesse.

\subsection{Monolithic suspension}

Fibers made of suitable materials, such as $\mathrm{SiO}_{2}$, and with high quality surfaces imply a low suspension thermal noise (Eq. (15)) and a higher resistance to breakage [12]. Moreover, reducing the diameter of these fibers (Fig. 2) assures a further reduction of the suspension thermal noise. To achieve this, we use the same method developed for Advanced Virgo in which two parts of silica, called anchors, are welded on a silica rod through a $\mathrm{CO}_{2}$ laser. Then, a long annealing is performed on the rod to reduce surface defects [22], and finally the fiber is produced [23]. Currently, there are two methods used to attach the fibers to the mirror:

- Virgo method: two parallel cuts (flats) are machined on the substrate barrel and polished with a $\lambda / 10$ flatness. Two silica supports (ears) are bonded to the flats, one per side, with the hydroxide-catalysis method [24,25]. Fibers are produced starting from silica rods, welded to silica parts (anchors) using a $\mathrm{CO}_{2}$ laser pulling machine. Then, the lower anchor of each fiber is glued to the lower side of the lateral support (ear), while the upper anchor is bonded to the top side of the steering stage (marionette) [23];

- LIGO method: as for the previous method, two lateral flats with a high quality surface are produced on the barrel of the mirror substrate and lateral supports are bonded with the hydroxide-catalysis method. Each support has two tips and the fibers are directly welded to those tips. In this way, the last gluing step foreseen by the Virgo method can be avoided [14].

Both methods show advantages and disadvantages. The main difference consists in the number of elements to be glued which implies a variation in thermal noise due to the bonding. We performed finite element analysis to estimate the effect on the small mirror $\left(10 \mathrm{~g}, 1^{\prime \prime}\right)$ thermal noise due to different thickness of the bonding layers in a Virgolike configuration. The results, summarised in Table 2 and shown in Figure 3, demonstrate that the bonded parts have a small effect on the total thermal noise calculated according to Levin's approach (see Sect. 4.4). Therefore, we decided to adopt a method similar to those developed for Virgo, which has the advantage of a better ease in the positioning of the fibers and guarantees a live control of the mirrors perpendicularity. Figure 3 also shows that the requirement for the quality of the surfaces to be glued is 
in line with an optical finish. This leads to a simplification of production and a cost reduction. Concerning the geometry of the silica fibers (mainly diameter and length), it is defined taking into account the evaluation described in Section 3.1.1.

\subsection{Double stage suspension thermal noise}

The thermal noise for the double pendulum system has been estimated by using the approach of the analytic model of the sequential pendula [26]. The double pendulum is composed of a first steering stage (marionette) plus the mirror as schematized in Figure 2. The equations of motion of the system give the impedance matrix $Z_{i j}(\omega)$ which takes into account all the dissipation processes present in the system $[27,28]$. Then, in order to calculate the thermal noise of the mirror suspension, which in our case corresponds to the element 2,2 of the impedance matrix, we have to apply the fluctuation dissipation theorem to the element 2,2 of the inverse of the impedance matrix [26]:

$$
X_{\text {therm }}(\omega)=\sqrt{\frac{4 k_{B} T}{\omega^{2}} \Re\left\{\left[Z^{-1}(\omega)\right]_{22}\right\}} .
$$

With a similar approach we estimate the transverse vibration modes of the suspension wire, which are called violin modes, in analogy with vibrating strings. Indeed, in suspension wires, when the tension due to the applied load dominates over their internal elasticity, they behave as vibrating strings. This effect, in terms of the power spectral density of the mirror displacement, can be modeled according to the approach made by Gonzáles and Saulson [29]:

$$
X_{\text {vio }}(\omega)=\sqrt{\frac{4 k_{B} T}{\omega} \frac{2 \rho r^{2} l}{\pi m^{2}} \sum_{n} \frac{\omega_{n}^{2} \phi_{n} / n}{\left(\omega_{n}^{2}-\omega^{2}\right)^{2}+\left(\omega_{n}^{2} \phi_{n}\right)^{2}}},
$$

where $r$ and $l$ are the fiber radius and length and $\omega_{n}$, $\phi_{n}$ represent the frequency and the total loss angle corresponding to the $n$-th harmonic. $\phi_{n}$ is defined as:

$$
\phi_{n}(\omega)=\frac{2 \phi(\omega)}{l k_{v}(\omega)}\left[1+\frac{(n \pi)^{2}}{\left.2 k_{v}(\omega)\right) l}\right]
$$

in which the wave number related to the flexural rigidity of the fiber is given by [29]:

$$
k_{v}(\omega)=\sqrt{\frac{\Lambda+\sqrt{\Lambda^{2}+4 Y J \rho \pi r^{2} \omega^{2}}}{2 Y J}},
$$

where $Y$ is the Young's modulus of the fiber and $J$ is the moment of inertia of the wire section with respect to its axis. In equation $(25), \phi(\omega)$ is the total loss angle given by the sum of the structural $\phi_{s}$, thermoelastic $\phi_{t e}(\omega)$ and surface losses $\phi_{e}$ :

$$
\phi(\omega)=\phi_{s}+\phi_{t e}(\omega)+\phi_{e} .
$$

Therefore, the total thermal noise of the double suspension system is given by the square of the quadratic sum of the
Table 2. The dissipative contribution due to the silicate bonding (i.e. the loss angle $\phi_{S B}$ ) is calculated for several values of thickness of the silicate layer to attach the ears to the mirror flats and to attach the lower anchors to the ears. Even increasing the thickness to few $\mu m$ the loss angle due to the silicate layers is still within the loss angle value of the high reflective coating estimated for a $10 \mathrm{~g}, 1^{\prime \prime}$ fused silica mirror which is $3.7 \times 10^{-6}$.

\begin{tabular}{lc}
\hline Thickness & $\phi_{S B}$ \\
\hline $315 \mathrm{~nm}$ & $4.0 \times 10^{-7}$ \\
$630 \mathrm{~nm}$ & $7.5 \times 10^{-7}$ \\
$1 \mu \mathrm{m}$ & $1.1 \times 10^{-6}$ \\
$2 \mu \mathrm{m}$ & $2.1 \times 10^{-6}$ \\
$2.5 \mu \mathrm{m}$ & $2.6 \times 10^{-6}$ \\
$3 \mu \mathrm{m}$ & $3.0 \times 10^{-6}$ \\
\hline
\end{tabular}

two contributions: thermal noise of the suspension and thermal noise of the violin modes:

$$
X_{\text {ThNS }}(\omega)=\sqrt{X_{\text {therm }}^{2}(\omega)+X_{v i o}^{2}(\omega)} .
$$

\subsubsection{Residual gas damping}

The residual gas damping due to Brownian motion of the air particles can introduce a non-negligible effect when considering the suspension thermal noise of a system like the one presented in this paper. Therefore, in our detailed thermal noise study, we must take into account this contribution in the overall calculation of the impedance matrix of the system. We suppose to work in high vacuum condition, having a residual gas pressure $p=10^{-9}$ mbar of molecular hydrogen. This can be expressed as a viscous term $\beta_{\mathrm{visc}}$ in the equation of motion of the mirror and then translated into a mechanical quality factor $Q_{\text {visc }}$ to be included in the total mechanical quality factor of the mirror in the impedance matrix of the system [36]. The viscous term can be expressed as $\beta_{\text {visc }}=p A / v_{T}$, where $p$ is the residual gas pressure $\left(10^{-9}\right.$ mbar in our case $), A$ is the section area of the mirror $A=\pi R^{2}$ and $v_{T}$ is the thermal velocity of the particles $v_{T}=\sqrt{k_{B} T / m_{H 2}}$ with $m_{H 2}$ the molecular mass of $H_{2}$ [36]. The viscous mechanical quality factor $Q_{\text {visc }}$ can be derived from the $\beta_{\text {visc }}$ according to $Q_{\text {visc }}=\omega_{0} m / \beta_{\text {visc }}$, where $\omega_{0}$ is the resonance of the suspended mirror of mass $m$. We evaluated the $Q_{\text {visc }}$ for both the end and input mirrors, obtaining the values: $Q_{\text {visc }, i}=2.75 \times 10^{9}$ and $Q_{\text {visc }, e}=8.35 \times 10^{8}$. In the noise budget plots for the two cases (Figs. 4 and 5) the dashed blue curves represent the thermal noise associated to the suspensions if we consider the contribute of the gas damping. These plots demonstrate the importance to satisfy the requirement to work in high vacuum conditions, for any experiment aiming to setting up an optical cavity like the one described in this work.

\subsection{Mirror thermal noise}

The mirror thermal noise, hereafter referred as ThNM, due to the reflective coating is calculated according to 


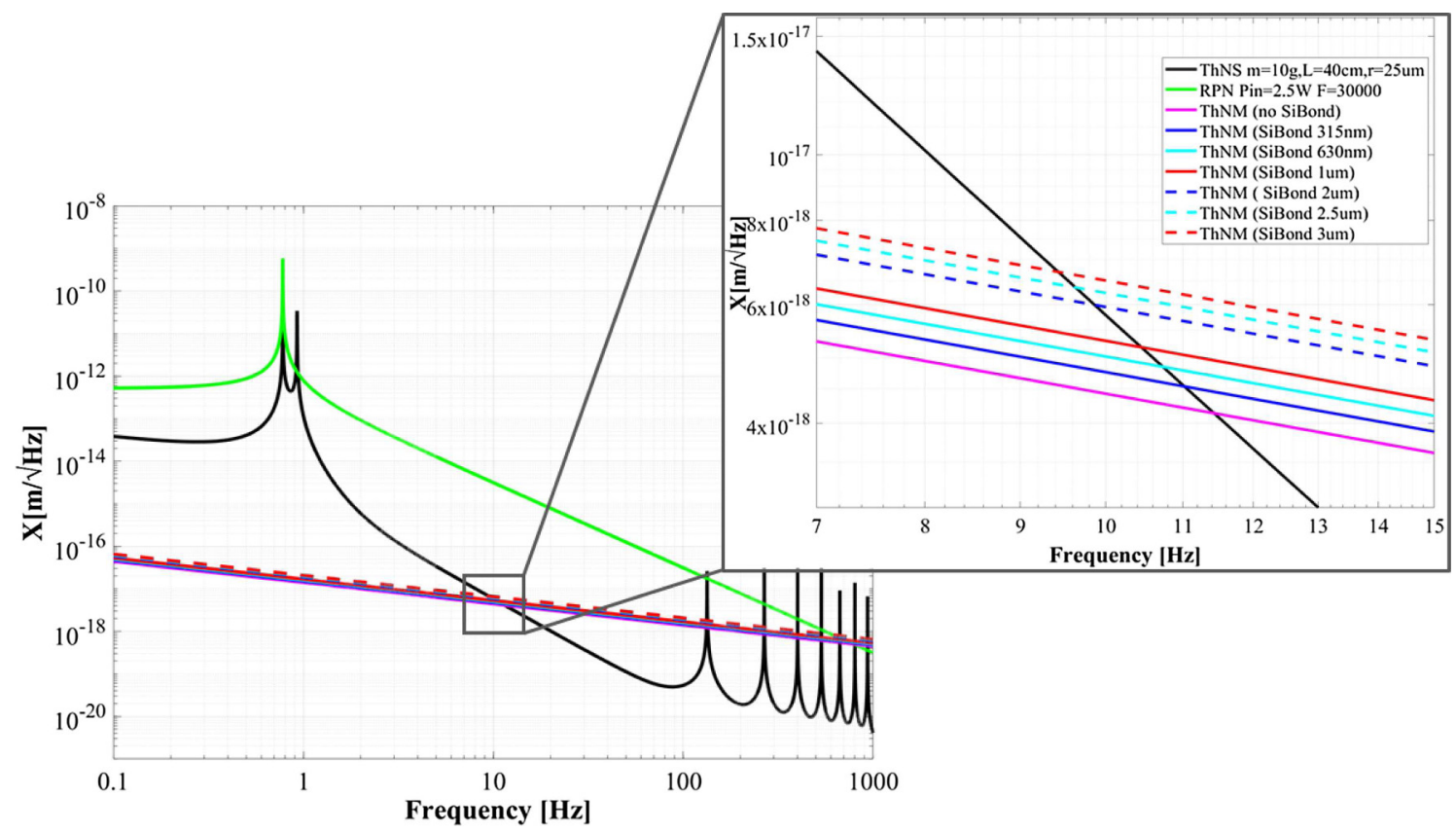

Fig. 3. The dissipative contribution due to the silicate bonding to the thermal noise (ThNM) of the lighter mirror $\left(1^{\prime \prime}, 10 \mathrm{~g}\right)$ is evaluated for several values of silicate layer thickness as explained in Table 2. Even increasing the thickness to few $\mu$ m the thermal noise of the mirror is still within the requirements.

Levin's approach [30] and using a finite element analysis (FEA) with ANSYS ${ }^{\circledR}$ software.

The mirror thermal noise, according to Levin's approach, is then given by:

$$
X_{\mathrm{ThNM}}(\omega)=\sqrt{\frac{8 k_{B} T}{\omega F_{0}^{2}} U_{\mathrm{mir}} \phi_{\mathrm{tot}}} .
$$

In equation (29), $U_{\text {mir }}$ is the total strain energy of the suspended mirror under an impinging Gaussian pressure:

$$
P(r)=\frac{2 F_{0}}{\pi w^{2}} e^{-\frac{2 r^{2}}{w^{2}}},
$$

given the integrated unitary force $F_{0}$, the beam waist $w$ of the laser on the mirror surface and the radial coordinate $r$ on the mirror surface. For the beam waist we set a value of $254 \mu \mathrm{m}$, which corresponds to $1 / 100$ of the $1^{\prime \prime}$ diameter mirror, and satisfies the stability condition for the optical cavity. All the dissipation processes are taken into account by the total loss angle, which is the sum of all the dissipative contributions calculated with the FEA following this formula:

$$
\phi_{\mathrm{tot}}=\phi_{B}+\sum_{\text {lay }} \frac{U_{\text {lay }}}{U_{\text {tot }}} \phi_{\text {mat }}^{\text {lay }}
$$

$\phi_{B}$ is the loss angle of the bulk substrate material and the second term represents the sum of all the loss angles related to the dissipative layers. For each of these layers, the loss angle of the material composing the layer $\phi_{\text {mat }}^{\text {lay }}$ is multiplied by the fraction of strain energy stored in the layer $U_{\text {lay }}$, over the total strain energy $U_{\text {tot }}$ stored in the system composed by the mirror substrate plus the dissipative layer under the Gaussian pressure of equation (30). $U_{\text {lay }}$ and $U_{\text {tot }}$ are evaluated through the FEA analysis. In the double pendulum model considered in this analysis, the dissipative layers are those related to the coating on the mirror intra-cavity surface and to the silicate bonding layers used to monolithically attach the ears to the mirrors and the lower anchors of the suspension wires to the ears (see Sect. 4.2). In order to reach a finesse in the range of some $10^{4}$, we consider coatings like those that can be produced via an ion beam sputtering (IBS) machine as described in Table 3 [35]. The $10 \mathrm{~g}$ end mirror (EM) and the $300 \mathrm{~g}$ input mirror (IM) coatings will be composed by alternative layers of low refraction index material (LR) and high refraction index material $(\mathrm{HR})$, made of $\mathrm{SiO}_{2}$ and $\mathrm{Ta}_{2} \mathrm{O}_{5}$ respectively. The thickness of these layers is different for the EM and IM, since the cavity end mirror should have a very low value for the transmittivity, while the input mirror a slightly higher one (see Tab. 3). For the silicate layers, related to the mirror monolithic suspension, we considered a thickness of $315 \mathrm{~nm}$, which is easily achievable applying the silicate bonding between the flats of the mirror lateral surface and the ears, and between the ears and the lower anchors of the silica fiber (see the Sect. 4.2). The intrinsic losses of the material composing the silicate bonding can be considered equal to $\phi_{\text {sibon }}=0.1$, while the density is the same of the bulk material $2200 \mathrm{~kg} / \mathrm{m}^{3}$ and the Young's modulus is $7.9 \mathrm{GPa}$. Therefore, we obtain:

$$
\phi_{\mathrm{tot}}=\phi_{B}+\frac{U_{\mathrm{coat}}^{H R}}{U_{\mathrm{tot}}} \phi_{\mathrm{mat}}^{H R}+\frac{U_{\mathrm{coat}}^{L R}}{U_{\mathrm{tot}}} \phi_{\mathrm{mat}}^{L R}+\frac{U_{S B}}{U_{\mathrm{tot}}} \phi_{S B}
$$

where the strain energy ratios for the coatings $\left(U_{\text {coat }}^{L R} / U_{\text {tot }}\right.$, $\left.U_{\text {coat }}^{H R} / U_{\text {tot }}\right)$ and for the silicate bonding $\left(U_{S B} / U_{\text {tot }}\right)$ lay- 


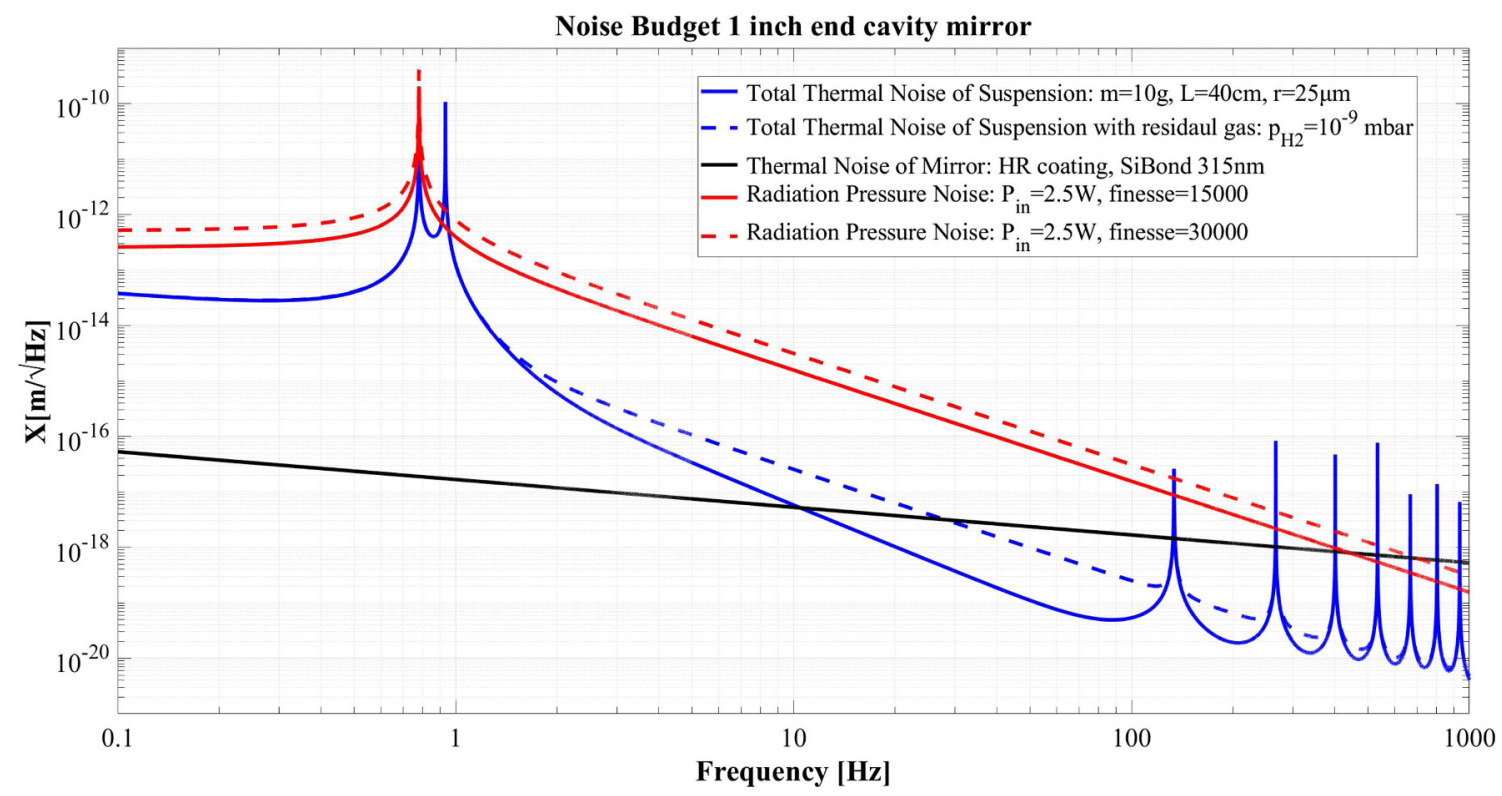

Fig. 4. Noise budget computed for the $1^{\prime \prime}$ diameter fused silica mirror of $10 \mathrm{~g}$ mass $(\mathrm{m})$ in double suspension configuration. The suspension wires are fused silica cylinders of $50 \mu \mathrm{m}$ diameter $(r=25 \mu \mathrm{m})$ and $40 \mathrm{~cm}$ length (l). The total suspension thermal noise (blue curve) considers also violin modes of the wires. The blue-dashed curve represents total suspension thermal noise when considering the effect of residual gas damping due to a pressure $p=10^{-9}$ mbar of molecular hydrogen. The mirror thermal noise (black curve) has been estimated with finite element analysis considering coating produced by a IBS machine [35] (see Tab. 3) and a $315 \mathrm{~nm}$ layer of silicate bonding to monolithically attach the fibers to the mirror. At $10 \mathrm{~Hz}$ the quantum radiation pressure noise for a finesse of 3000 is a factor 600 above the thermal noise.

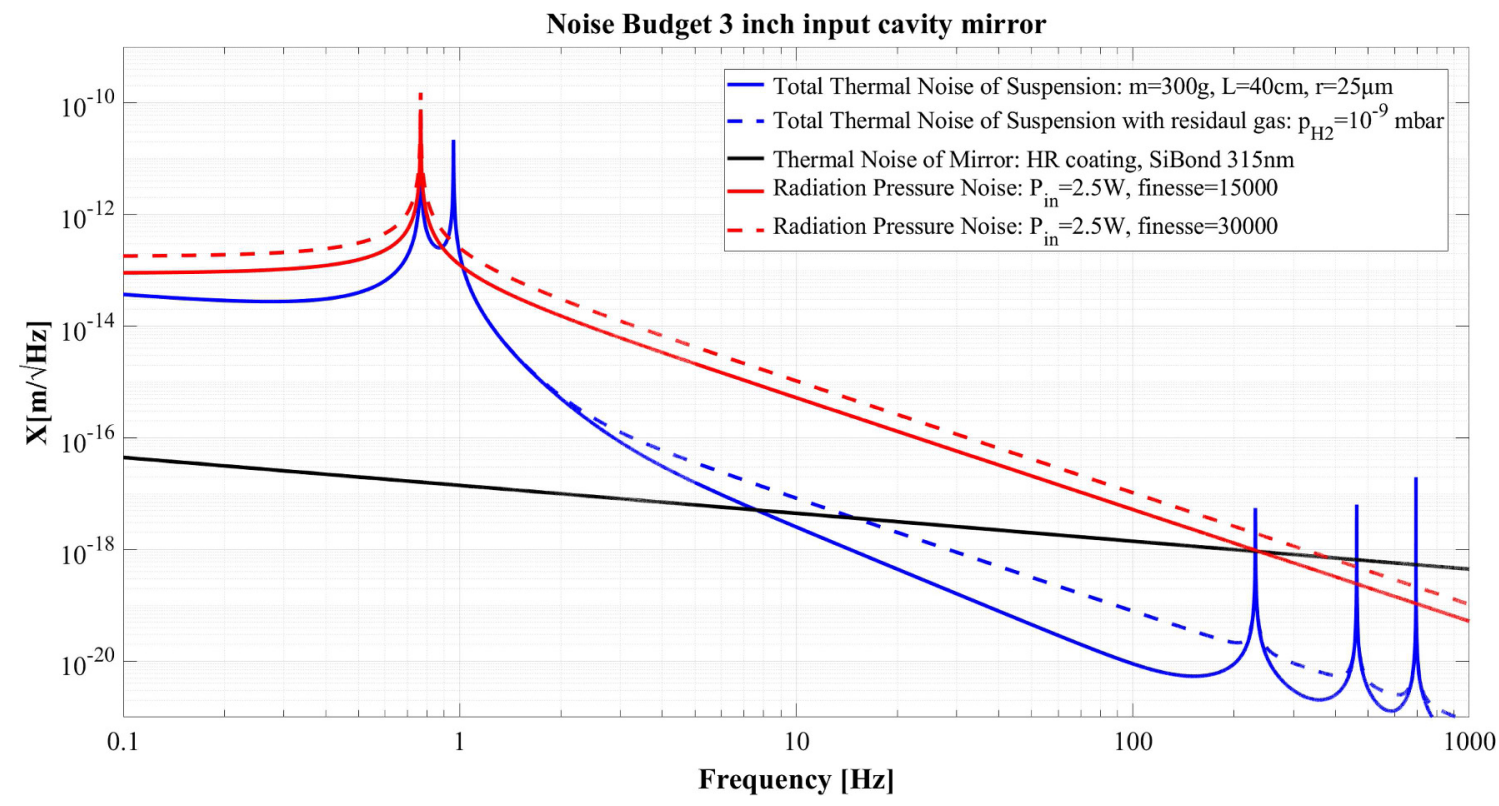

Fig. 5. Noise budget computed for the $3^{\prime \prime}$ diameter fused silica mirror of $300 \mathrm{~g}$ mass (m) in double suspension configuration. The suspension wires are $\mathrm{SiO}_{2}$ cylinders of $50 \mu \mathrm{m}$ diameter $(2 r)$ and $40 \mathrm{~cm}$ length (l). The total suspension thermal noise (blue curve) considers also those related to violin modes of the wires. The blue-dashed curve represents total suspension thermal noise when considering the effect of residual gas damping due to a pressure $p=10^{-9}$ mbar of molecular hydrogen. The mirror thermal noise (black curve) has been estimated with finite element analysis considering coating produced by a IBS machine [35] (see Tab. 3) and a $315 \mathrm{~nm}$ layer of silicate bonding to monolithically attach the fibers to the mirror. 
Table 3. Parameters used in our analysis for the coating layers of the Fabry-Pérot cavity end mirror (EM) and input mirror (IM). We considered coatings made of layers of high refraction index (HR) and low refraction index (LR) materials, produced with a SPECTOR 500 machine according to [35].

\begin{tabular}{lcc}
\hline Coating & HR index & LR index \\
\hline Material & $\mathrm{Ta}_{2} \mathrm{O}_{5}$ & $\mathrm{SiO}_{2}$ \\
Density & $7470 \mathrm{~kg} / \mathrm{m}^{3}$ & $2360 \mathrm{~kg} / \mathrm{m}^{3}$ \\
Young's modulus & $121 \mathrm{GPa}$ & $78 \mathrm{GPa}$ \\
Loss angle & $1.9 \times 10^{-4}$ & $1.5 \times 10^{-4}$ \\
Thickness IM & $1.995 \mu \mathrm{m}$ & $2.653 \mu \mathrm{m}$ \\
Thickness EM & $2.673 \mu \mathrm{m}$ & $3.980 \mu \mathrm{m}$ \\
\hline
\end{tabular}

ers are calculated with FEA simulations. We assume here that the coatings can withstand the high power stored in the cavity. This condition must be verified with the coating producer when setting up an experiment using the described configuration.

\subsubsection{Thermal noise budget for the end mirror}

A suspended mirror in a Fabry-Prot cavity, in order to be sensitive to the radiation pressure fluctuations, should have a spectral density of the mirror displacement due to the quantum radiation pressure noise at least one order of magnitude above those ones due to the thermal noises. According to the methods explained in Sections 4.3 and 4.4, we evaluate now the total noise budget for $10 \mathrm{~g}$ mass, $1^{\prime \prime}$ diameter, monolithically suspended silica mirror. In this analysis, we consider the $10 \mathrm{~g}$ mirror as the end mirror of a Fabry-Pérot cavity having a high reflective coating like the one produced by IBS technique and described in Table 3 [35]. The suspension system considered is a double stage, as shown in the scheme of Figure 2, and the thermal noise of the suspension is calculated using equation (28). The mirror thermal noise is estimated according to Levin's approach and by evaluating all the dissipative contributions through finite element analysis as explained in Section 4.4. The total loss angle, calculated according to equation (32), is then used to compute the mirror thermal noise which is represented by the black curve in Figure 4. The noise budget estimation of Figure 4 shows that the cavity realized suspending a mirror with mass $10 \mathrm{~g}$ will be quantum radiation pressure noise-limited in the audio-frequency band of ground based gravitational wave detectors below $1 \mathrm{kHz}$. In particular at $10 \mathrm{~Hz}$, given an input laser power $P_{\text {in }}=2.5 \mathrm{~W}$ and cavity finesse $\mathcal{F}=3 \times 10^{4}$, the quantum radiation pressure noise $(\mathrm{RPN})$ is about $3 \times 10^{-15} \mathrm{~m} / \sqrt{\mathrm{Hz}}$ while the thermal noise due to the mirror and the suspensions are about $5 \times 10^{-18} \mathrm{~m} / \sqrt{\mathrm{Hz}}$ : a factor 600 lower than radiation pressure noise. As shown in the blue-dashed curve in Figure 4, also considering the effect of a residual gas pressure $10^{-9}$ mbar of molecular hydrogen, which means working in high vacuum conditions, the suspension thermal noise still satisfies the requirement.

\subsubsection{Thermal noise budget for the input mirror}

We repeat the analysis done on the lighter mirror, for a heavier mirror of mass $300 \mathrm{~g}$ and diameter $3^{\prime \prime}$. We chose a mirror of mass $300 \mathrm{~g}$, value still compliant with the requirements described in the previous analysis and which corresponds to an off-the-shelf bulk fused silica mirror having 3 times the diameter and the thickness of the lighter one (i.e. $3^{\prime \prime}$ diameter and $3 \mathrm{~cm}$ thickness). The procedure used for the estimation of the total suspension thermal noise, the mirror thermal noise and the quantum radiation pressure noise is the same used for the $10 \mathrm{~g}$ mirror. In this analysis, we consider the $300 \mathrm{~g}$ mirror as the input mirror of the Fabry-Pérot cavity, with a partially transmitting coating that can be produced with an IBS machine whose parameters are described in Table 3 [35]. In this section, Figure 5 showcases the estimated noises for this $300 \mathrm{~g}$ (or equivalently $3^{\prime \prime}$ ) monolithically suspended mirror. The noise budget estimations of Figures 4 and 5 show that the cavity realized suspending an end mirror with mass $10 \mathrm{~g}$ and an input mirror with mass $300 \mathrm{~g}$ will be quantum radiation pressure noise-limited in the audio-frequency band of ground based gravitational wave detectors below $1 \mathrm{kHz}$. Obviously, this is true if we don't take into account the power and phase fluctuations produced by the laser source itself.

\section{Other sources of noise}

In this work we limited our analysis to the thermal noise associated with the proposed tabletop optical cavity. Nevertheless, other sources of noise must be taken into account to be able to observe and exploit the effect of quantum radiation pressure noise inside the cavity composed of double-stage suspended mirrors. In particular, setting up a dedicated experiment, one should consider a system capable of significant seismic noise attenuation but also to cancel out the noises induced by the laser source inside the cavity.

\subsection{Seismic noise attenuation}

The seismic noise can be attenuated by suspending the optical cavity bench from the last steering filter of a Superattenuator (a chain of mechanical filters plus an inverted pendulum) like those used for Virgo test masses [10,11], which ensures about $180 \mathrm{~dB}$ of seismic noise attenuation at $10 \mathrm{~Hz}$. An alternative method to filter out the seismic noise relies on the use of active damping systems, such those used for the test masses of LIGO [31]. In this way the ground motion would not limit the possibility to observe the quantum radiation pressure effect in the cavity in the band of frequency considered in this work (few $\mathrm{Hz}-$ hundreds of $\mathrm{Hz}$ ).

\subsection{Interferometric configuration}

As pointed out at the end of Section 4, the relative intensity noise (RIN) and the phase fluctuations of the laser 
source could spoil the low thermal noise design of the proposed double-stage suspended optical cavity. A solution to this important issue is to combine two identical FabryPérot cavities, like the one presented in this paper and depicted in Figure 2, with a 50/50 beam splitter to form the arm cavities of a Michelson interferometer. In this way it is possible to cancel out the common-mode noises due to the laser stability in power and phase. Of course, the balancing of the beam splitter would be crucial to achieve a good common-mode rejection ratio. Another advantage of the interferometric configuration is that it would be capable of producing squeezed light of vacuum states through the ponderomotive mechanism within the detection band of ground-based gravitational wave interferometers.

\section{Conclusions}

The study presented in this paper demonstrates that it is possible to realise tabletop optical cavities with $10 \mathrm{~g}-$ $300 \mathrm{~g}$ scale mirrors suspended in quantum radiation pressure noise regime. According to our estimations, adopting a suitable suspension system, composed of a double pendulum with a monolithic suspension and working in high vacuum condition, the suspension thermal noise is lower than the quantum radiation pressure noise, e.g. of a factor 600 at $10 \mathrm{~Hz}$. Moreover, the use of high reflective coatings, like those achievable with a IBS coating machine providing the parameters of Table 3, allows to keep the mirror thermal noise low enough to make the radiation pressure noise observable. Note that in this context we have assumed that the coatings can withstand the high power stored in the cavity. This requirement is of paramount importance and when setting up the experiment it should be verified with the coating producer. Combining two identical cavities like those ones studied in this work would result in a tabletop interferometer capable of ponderomotive squeezing in the typical frequency band of ground-based gravitational wave interferometers. Another interesting application of the interferometric configuration of the studied cavities is to use the proposed setup as a demonstrator of the Einstein-Podolsky-Rosen (EPR) technique [32] to generate frequency-dependent squeezing. Indeed, before a possible application to GW detectors, like Advanced Virgo, the EPR squeezing technique needs to be properly tested with a RPN-limited interferometer. This can be achieved by integrating a dedicated EPR squeezing experiment, with the monolithically suspended high finesse optical cavities, here proposed, as described in [33,34]. We can conclude that, exploiting the ponderomotive technique, or testing the EPR squeezing technique, a quantum radiation pressure noise-limited interferometer, obtained from the combination of two monolithically suspended high finesse optical cavities, like those described in this study, can be used to achieve broadband quantum noise reduction in GW detectors below $1 \mathrm{kHz}$.

Open Access funding provided by Università degli Studi di Roma La Sapienza within the CRUICARE Agreement. We want to acknowledge the institutions which funded this study: the Italian MIUR, in the framework of the PRIN PPPS, in particular for the initial R\&D, and the INFN - CSN5, in the framework of the SIPS experiment. We want to thank all the people who contributed to this study in the INFN branches of Rome, Naples, Perugia and Pisa, and the European Gravitational Observatory (EGO) for the infrastructure and technical support. A special thank goes to E. Calloni for the initial concept of design and preliminary noise budget estimation, as well as P. Puppo for the useful advice for developing the thermal noise model, F. Piergiovanni and M. Montani for the feasibility study and test of thin silica fibers like those considered in this study.

\section{Author contribution statement}

All authors actively contributed to the realisation of the study presented in this paper. Conceptualization: M.D.L., S.D.P., L.N.; modeling and investigation: S.D.P., L.N.; mirror suspension design: F.T., L.N., S.D.P. All authors contributed writing this work, and agreed to the published version of the manuscript.

Publisher's Note The EPJ Publishers remain neutral with regard to jurisdictional claims in published maps and institutional affiliations.

Open Access This is an open access article distributed under the terms of the Creative Commons Attribution License (https://creativecommons.org/licenses/by/4.0/), which permits unrestricted use, distribution, and reproduction in any medium, provided the original work is properly cited.

\section{References}

1. F. Acernese et al., Class. Quantum Grav. 32, 024001 (2015)

2. F. Acernese et al., Phys. Rev. Lett. 125, 131101 (2020)

3. H. Yu et al., submitted to Nature (2020)

4. T. Corbitt, N. Mavalvala, J. Opt. B 6, S675 (2004)

5. V.B. Braginsky, F.Y. Khalili, Rev. Mod. Phys. 68, 1 (1996)

6. B.S Sheard, M.B. Gray, M. Mow-Lowry, D.E. McClelland, S.E. Whitcomb, Phys. Rev. A 69, 051801 (2004)

7. T. Corbitt et al., Phys. Rev. A 73, 023801 (2006)

8. T.P. Purdy, P.L. Yu, R.W. Peterson, N.S. Kampel, C.A. Regal, Phys. Rev. X 3, 031012 (2013)

9. N. Aggarwal, N. Mavalvala, T. Corbitt et al., Nature 568, 364 (2019)

10. T. Accadia et al., J. Low Freq. Noise V. A. 63, 30 (2011)

11. S. Braccini et al., Astropart. Phys. 23, 557 (2005)

12. P. Amico et al., Class. Quantum Grav. 19, 1669 (2002)

13. D. Aisa et al., Nucl. Instrum. Methods A 824, 644 (2016)

14. A.V. Cumming et al., Class. Quantum Grav. 29, 035003 (2012)

15. G. Cagnoli et al., Phys. Lett. A 272, 39 (2000)

16. R. Kubo, Rep. Prog. Phys. 1 (Part I) 29, 255 (1966)

17. M. Lorenzini et al., Class. Quantum Grav. 27, 084021 (2010)

18. A.M. Gretarsson, G.M. Harry, Rev. Sci. Instrum. 70, 10 (1999)

19. A.M. Gretarsson et al., Rev. Sci. Instrum. 77, 023906 (2006) 
20. A.M. Gretarsson et al., Phys. Lett. A 270, 108 (2000)

21. S. Di Pace, Ph.D. Thesis, 2014, https://tel.archivesouvertes.fr/tel-01170076/file/2014NICE4108.pdf

22. A. Heptonstall et al., Class. Quantum Grav. 31, 105006 (2014)

23. F. Travasso et al., J. Phys.: Conf. Ser. 957, 012012 (2018)

24. D.H. Gwo, 2001 US Patent No US 6284085 B1, 2001

25. D.H. Gwo, 2003 US Patent No US 6548176 B1, 2003

26. E. Majorana, Y. Ogawa, Phys. Lett. A 233, 162 (1997)

27. R.F. Green, H.B. Callen, Phys. Rev. 83, 1231 (1951)

28. H.B. Callen, R.F. Green, Phys. Rev. 88, 1387 (1952)
29. G.I. González, P.R. Saulson, J. Acoust. Soc. Am. 96, 207 (1994)

30. Y. Levin, Phys. Rev. D 57, 659 (1998)

31. F. Matichard et al., Class. Quantum Grav. 32, 185003 (2015)

32. Y. Ma et al., Nature 13, 776 (2017)

33. V. Sequino et al., in GRASS 2019 Proceeding (2020), http: //dx.doi.org/10.5281/zenodo. 3554320

34. S. Di Pace et al., in GRASS 2019 proceeding (2020), http: //dx.doi.org/10.5281/zenodo. 3569196

35. M. Granata et al., Class. Quantum Grav. 37, 095004 (2020)

36. A. Cavalleri et al., Phys. Lett. A 374, 3365 (2010) 